US-97-06

Febrary,1998(revised)

hep-th/9707025

\title{
Weyl Anomaly of 2D Dilaton-Scalar Gravity and Hermiticity of System Operator
}

\author{
Shoichi ICHINOSE 円 \\ Department of Physics, University of Shizuoka, \\ Yada 52-1, Shizuoka 422, Japan.
}

\begin{abstract}
Weyl(Conformal) anomaly in the dilaton-scalar system in 2 dimensional gravity is examined. We take the heat-kernel regularization for the ultraviolet divergences. Generally the Weyl anomaly is determined by the 2nd order differetial (elliptic) operator of the system and the definition of the measure. We have the freedom of the operator choice caused by the arbitrariness of total divergences (surface terms) in the action. We focus on such points and the hermiticity of the system operator.
\end{abstract}

PACS NO: 11.10.-z, 04.60.K, 11.30.-j

Keywords: 2 dimensional dilaton gravity, Weyl anomaly, Anomaly formula

${ }^{1}$ E-mail address: ichinose@u-shizuoka-ken.ac.jp 


\section{Introduction}

Motivated by 2 dim or 4 dim black hole physics [1], Bousso and Hawking[2] recently have calculated the Weyl anomaly for the dilaton coupled scalar theory in 2 dim gravity.

$$
S_{m}=\frac{1}{2} \int d^{2} x \sqrt{g} \mathrm{e}^{-2 \phi} g^{\mu \nu} \nabla_{\mu} f \cdot \nabla_{\nu} f
$$

where $g_{\mu \nu}, f$ and $\phi$ are the metric, a scalar matter field and the dilaton respectively. It is invariant under the Weyl transformation.

$$
\phi^{\prime}=\phi \quad, \quad f^{\prime}=f \quad, \quad g_{\mu \nu}^{\prime}=e^{2 \alpha(x)} g_{\mu \nu} \quad .
$$

They have obtained, using the generalized zeta function, the Weyl anomaly due to the scalar matter quantum effect ( $g_{\mu \nu}$ and $\phi$ are background fields ), as

$$
\begin{array}{r}
T=\sqrt{g}\left(q_{1} R+q_{2}(\nabla \phi)^{2}+q_{3} \nabla^{2} \phi\right), \\
q_{1}=-\frac{1}{24 \pi}, \quad q_{2}=-\frac{1}{4 \pi}, \quad q_{3}=-\frac{1}{12 \pi} .
\end{array}
$$

They pointed out, in the induced effective action derived from the above result, the term $-2 \phi R$ naturally appears from the $q_{3}$ term of eq.(3). The term was originally itroduced by hand in [3] or from considerations of (Weyl) anomalies in [由]. The result shows an important role of the dilaton field. Stimulated by this work, some authors have examined the problem in different ways. The divergence structure of a wide class of theories, which includes the above model, was already examined by [5] in the dimensional regularization. Based on the work, Ref.[6] has calculated the Weyl anomaly of (1). Their result differs from eq.(3) in $q_{3}=1 / 4 \pi$. They have also obtained it for a wide class of dilaton coupled scalar theories taking into account not only the scalar matter but also the dilaton quantum effect. Ref. [7, 8] has also examined the Weyl anomaly taking into account all fields (graviton, dilaton and scalar matter) quantum effects.

It is true that the dilaton field is becoming more and more important in accord with the rapid progress of the string theory. The string theory predicts very naturally the existence of the dilaton in its low energy (field theory) limit. We do not have much experience about the correct treatment of the dilaton field and its characteristic interaction $\exp (-2 \phi)$. In this sense, the close analysis of the present problem looks important.

Some problems in the Weyl anomaly itself also motivate us to do the present analysis. Compared with the chiral anomaly ( anomaly related to " $\gamma_{5}$ ") the general argument of Weyl anomaly is not so easy [9, 10, 11, 12]. It originates partly from the difficulty of clear characterization of Weyl-anomaly terms by a simple symmetry argument. The Weyl anomaly

looks to be more influenced by the local dynamics and generally requires more complicated calculation than the chiral one. The present 2 dim model is a nice simple model, by which we can test some general "belief" about the Weyl anomaly. To say it more concretely, we focus on the following points: 1) General structure of the anomaly terms; 2) Regularization 
dependence of the Weyl anomaly; 3) The origin of the discrepancy in the value of $q_{3}$; 4) How the arbitrariness of the total divergence affects the Weyl anomaly. The quantization of the scalar field $f$ is formally defined by the path-integral.

$$
\exp \Gamma[g, \phi]=\int d \mu(f ; g, \phi) \exp S_{m}[f ; g, \phi],
$$

where $d \mu(f ; g, \phi)$ is the integration measure and requires the precise definition. which will become a key point in later analysis.

There are several ways of obtaining the Weyl anomaly: 1) the generalized zeta function ([2]); 2) the counterterm formula expressed in the dimensional regularization( [6]); 3) the heat-kernel regularization( [13]). We calculate the Weyl anomaly in the approach 3) and take the Fujikawa's standpoint [14, 15] about the anomaly. Compared with the counter-term calculation with the dimensional regularization, the advantage of the present approach is that, once the differential operator of the system (we call it system operator) is fixed, we have no ambiguity, in all stages of calculation, arising from the total divergences [16]. This is because the heat-kernel approach deals with the operator directly, not with the (effective) action. Ref. [6] hints that the discrepancy could come from total divergence ambiguity in the counterterm calculation. We will clarify this point in this paper. Ref. [6] has also pointed out that the way of fixing $q_{3}$ which ref. [2] has taken, is not unique because of the presence of ambiguity in the procedure of translating the Weyl anomaly result to the induced (nonlocal) action.

Before a detailed analysis, we fix the general form of the Weyl anomaly by the symmetry arguments. Generally the anomaly is caused by a conflict between some symmetries. In the present case, they are the ( $2 \mathrm{dim}$ ) general coordinate invariance (diffeomorphism) and the Weyl symmetry. We take the familiar convention of keeping the general coordinate invariance. Because of the quantization effect, the system cannot keep the Weyl symmetry. The general form is obtained as (3) with general values of $q$ 's by the following requirements: 1) $2 \mathrm{dim}$ general coordinate invariance,; 2) Weyl invariance (up to total divergences) 9, 10, 11, 12,; 3) Invariance under the constant shift of the dilaton field, $\phi \rightarrow \phi+$ const.. The requirement 3 ) is necessary because the constant shift changes the action (1) only by an overall constant factor, which can be absorbed into a constant rescaling of the scalar $f$. Without the condition 3 ) we would have the arbitrariness of functionals $A(\phi)$ and $B(\phi)$ in two terms, $A(\phi)(\nabla \phi)^{2}$ and $B(\phi) \nabla^{2} \phi$, as the anomaly general form [17]. Among the three terms, $q_{3}$-term is a "trivial term" in the terminology of the anomaly-cohomology because the (infinitesimal) Weyl transformation of the local term $\sqrt{g} \phi R$ shows

$$
(\sqrt{g} \phi R)^{\prime}=\sqrt{g}\left(\phi R+2 \alpha(x) \cdot \nabla^{2} \phi\right)+\text { total-derivative }+O\left(\alpha^{2}\right) \quad,|\alpha| \ll 1 \quad .
$$




\section{Anomaly formula and hermiticity of system operator}

First we rescale the scalar field, $f$, in order to adjust the kinetic term in the ordinary form: $f \exp (-\phi)=F$. Then eq.(1) is equivalent to, up to a total derivative,

$$
\begin{gathered}
S_{m}^{1}=\frac{1}{2} \int d^{2} x \sqrt[4]{g} F \vec{D}_{1}(\sqrt[4]{g} F) \\
=S_{m}-\frac{1}{2} \int d^{2} x \sqrt{g} \nabla_{\mu}\left(F \nabla^{\mu} F+a \nabla^{\mu} \phi \cdot F^{2}\right) \\
\vec{D}_{1}=\sqrt[4]{g}\left\{-\nabla^{2}+2(1-a) \nabla_{\mu} \phi \cdot \nabla^{\mu}-a \nabla^{2} \phi+\nabla_{\mu} \phi \cdot \nabla^{\mu} \phi\right\} \frac{1}{\sqrt[4]{g}},
\end{gathered}
$$

where a free parameter, $a$, appears in the first derivative term. It represents the freedom of the total derivative terms. A purpose of this section is to check the effect of the freedom. We can take the BRS-invariant measure for the matter scalar field by taking $\tilde{F}=\sqrt[4]{g} F$ instead of $F[18]$. This is the reason for the factors $\sqrt[4]{g}, \frac{1}{\sqrt[4]{g}}$ in $\vec{D}_{1}$ above. The 2nd order differential operator $\vec{D}_{1}$ is hermitian for $a=1$, when we define the inner product and the hermitian conjugate as

$$
\begin{gathered}
<\tilde{F}_{1} \mid \tilde{F}_{2}>=\int d^{2} x \tilde{F}_{1}^{\dagger} \tilde{F}_{2}=\int d^{2} x \sqrt{g} F_{1}^{\dagger} F_{2}=\int d^{2} x \sqrt{g} \mathrm{e}^{-2 \phi} f_{1}^{\dagger} f_{2} \\
<\vec{D}_{1} \tilde{F}_{1}\left|\tilde{F}_{2}>=<\tilde{F}_{1}\right| \vec{D}_{1}^{\dagger} \tilde{F}_{2}>.
\end{gathered}
$$

This choice is most natural for the operator $\vec{D}_{1}$. For the case of $a \neq 1, \vec{D}_{1}$ is not hermitian. Note here that the factor of $\mathrm{e}^{-2 \phi}$ appears in the inner product. This will be compared with the case of Sec.3.

The above definition of the inner product between the fields $\tilde{F}$ 's fixes the integration measure in the path integral as follows 14. Let $\left\{u_{n}(x)\right\}$ be the complete and orthogonal set of the eigen functions of the operator $\vec{D}_{1}$.

$$
\vec{D}_{1} u_{n}=\lambda_{n} u_{n} \quad, \quad<u_{n} \mid u_{m}>=\int d^{2} x u_{n}^{\dagger} u_{m}=\delta_{n m} .
$$

From the completeness, a general field $\tilde{F}=\sqrt[4]{g} F$ can be expressed as

$$
\tilde{F}=\sum_{n} c_{n} u_{n} \quad, \quad c_{n} \equiv<u_{n} \mid \tilde{F}>
$$

where the orthogonality is used. Then the integration measure in the path-integral can be precisely defined as

$$
\begin{gathered}
\exp \Gamma_{1}[g, \phi]=\int d \mu_{1}(f ; g, \phi) \exp S_{m}^{1}[f ; g, \phi] \quad, \quad S_{m}^{1}=\frac{1}{2} \int d^{2} x \tilde{F} \vec{D}_{1} \tilde{F} \\
d \mu_{1}(f ; g, \phi)=\prod_{n} d c_{n} \equiv \mathcal{D} \tilde{F}
\end{gathered}
$$

This measure $d \mu_{1}$ depends on $a$ because $u_{n}$, by which the expansion coefficients $c_{n}$ is obtained, depends on $a$ through the eigen value equation (8). 
Following Fujikawa, let us evaluate the Jacobian $J$ in the measure change due to Weyl transformation (2). We regard the Jacobian as the Weyl anomaly.

$$
\begin{gathered}
\tilde{F}^{\prime} \equiv \sum_{n} c_{n}^{\prime} u_{n}=\mathrm{e}^{\alpha(x)} \tilde{F}(x)=\sum_{n} c_{n} \mathrm{e}^{\alpha(x)} u_{n} \\
c_{n}^{\prime}=\sum_{m} c_{m}<u_{n}\left|\mathrm{e}^{\alpha(x)} u_{m}>=c_{n}+\sum_{m} c_{m}<u_{n}\right| \alpha(x) u_{m}>+\mathrm{O}\left(\alpha^{2}\right) \\
J=\operatorname{det}\left(\frac{\partial c_{n}^{\prime}}{\partial c_{m}}\right)=\exp \operatorname{Tr} \ln \left(\delta_{m n}+<u_{n} \mid \alpha(x) u_{m}>+\mathrm{O}\left(\alpha^{2}\right)\right) \\
=\exp \left[\int d^{2} x \alpha(x) \sum_{n} u_{n}^{\dagger}(x) u_{n}(x)+\mathrm{O}\left(\alpha^{2}\right)\right]
\end{gathered}
$$

Therefore the Weyl anomaly is obtained as

$$
T_{1}=\left.\frac{\delta J}{\delta \alpha(x)}\right|_{\alpha=0}=\sum_{n} u_{n}^{\dagger}(x) u_{n}(x)
$$

This expression is generally divergent, and must be regularized. We take the heat-kernel regularization as

$$
\begin{gathered}
T_{1}=\lim _{t \rightarrow+0} \sum_{n} u_{n}^{\dagger}(x) u_{n}(x) \mathrm{e}^{-\lambda_{n} t}=\lim _{t \rightarrow+0} \operatorname{tr} G_{1}(x, x ; t) \\
G_{1}(x, y ; t) \equiv \sum_{n} u_{n}(x) \mathrm{e}^{-\lambda_{n} t} u_{n}^{\dagger}(y)
\end{gathered}
$$

$G_{1}(x, y ; t)$ satisfies the heat equation (15) below with $\vec{D}=\vec{D}_{1}$. For the case $a=1, \vec{D}_{1}$ is hermitian and we can write $T_{1}$ and $G_{1}(x, y ; t)$ as

$$
\begin{gathered}
T_{1}=\lim _{t \rightarrow+0} \sum_{n} u_{n}^{\dagger}(x) u_{n}(x) \mathrm{e}^{-\lambda_{n} t}=\lim _{t \rightarrow+0} \sum_{n}<n\left|x>\mathrm{e}^{-\vec{D}_{1} t}<x\right| n> \\
=\lim _{t \rightarrow+0} \sum_{n} \operatorname{tr}<x\left|n>\mathrm{e}^{-\vec{D}_{1} t}<n\right| x>=\lim _{t \rightarrow+0} \operatorname{tr}<x\left|\mathrm{e}^{-\vec{D}_{1} t}\right| x>=\lim _{t \rightarrow+0} \operatorname{tr} G_{1}(x, x ; t) \\
G_{1}(x, y ; t) \equiv<x\left|\mathrm{e}^{-\vec{D}_{1} t}\right| y>
\end{gathered}
$$

We note some general facts about the heat-kernel $G(x, y ; t)[13$. It is rigorously defined by the heat equation in terms of an 2 nd order differential operator $\vec{D}$,

$$
\left(\frac{\partial}{\partial t}+\vec{D}\right) \quad G(x, y ; t)=0
$$

and can be generally solved perturbatively even for the non-hermitian operator under the condition that the operator $\vec{D}$ asymptotically goes to $-\delta_{\mu \nu} \partial_{\mu} \partial_{\nu}$ in the weak field limit [13]. In the present model, the weak field means $g_{\mu \nu}=\delta_{\mu \nu}+h_{\mu \nu},\left|h_{\mu \nu}\right| \ll 1,|\phi| \ll 1$. We present a useful anomaly formula valid for most 2 dim gravity-matter theories. For a general theory, specified by general background fields $\mathcal{N}_{\mu}$ and $\mathcal{M}$ ( which are general covariants) as below, the Weyl anomaly $T$ is given by

$$
\begin{array}{r}
\vec{D}=\sqrt[4]{g}\left(-\nabla^{2}-\mathcal{N}_{\mu} \nabla^{\mu}-\mathcal{M}\right) \frac{1}{\sqrt[4]{g}}, \\
T=\left.\operatorname{tr} G(x, x ; t)\right|_{t^{0}}=\frac{1}{4 \pi} \sqrt{g} \operatorname{Tr}\left(-\frac{1}{6} R+\mathcal{M}-\frac{1}{2} \nabla_{\mu} \mathcal{N}^{\mu}\right) .
\end{array}
$$


"Tr" in the above equation means "trace" over all field indices: $i, j=1,2, \cdots, N ; \operatorname{Tr} \mathcal{M}=$ $\mathcal{M}^{i i}$, etc. This formula is easily derived from two facts: 1) the formula must be general invariant, 2) for the weak field gravity case, it leads to eq.(27) of ref. [13] or eq.(40) of ref. [19].

In the present special case of $\vec{D}_{1}\left(\mathcal{N}_{\mu}=-2(1-a) \nabla_{\mu} \phi, \mathcal{M}=a \nabla^{2} \phi-\nabla_{\mu} \phi \cdot \nabla^{\mu} \phi, N=1\right)$, we obtain the Weyl anomaly as

$$
\begin{gathered}
T_{1}=\frac{1}{4 \pi} \sqrt{g}\left(-\frac{1}{6} R-\nabla_{\mu} \phi \cdot \nabla^{\mu} \phi+\nabla^{2} \phi\right), \\
q_{1}=-\frac{1}{24 \pi} \quad, \quad q_{2}=-\frac{1}{4 \pi} \quad, \quad q_{3}=\frac{1}{4 \pi} .
\end{gathered}
$$

The final result does not depend on the free parameter $a$ and agrees with that of Ref. [6] .20] The result of $a$-independence say the arbitrariness of total derivative terms in the first derivative does not affect the Weyl anomaly.

\section{Second Choice of Measure}

The dilaton-scalar theory (1) can be modified to, up to total divergences,

$$
\begin{aligned}
S_{m}^{2}= & \frac{1}{2} \int d^{2} x \sqrt{g} \mathrm{e}^{-2 \phi} f\left(-\nabla^{2}+2 \nabla_{\mu} \phi \cdot \nabla^{\mu}\right) f \\
= & S_{m}+\frac{1}{2} \int d^{2} x \sqrt{g} \nabla^{\nu}\left(\mathrm{e}^{-2 \phi} f \nabla_{\nu} f\right) .
\end{aligned}
$$

$S_{m}^{2}$ seems to give the same Weyl anomaly as $S_{m}^{1}$ if the situation is the same as in Sect.2. In this section we take the following one

$$
\vec{D}_{2} \equiv \sqrt[4]{g} \mathrm{e}^{-2 \phi}\left(-\nabla^{2}+2 \nabla_{\mu} \phi \cdot \nabla^{\mu}\right) \frac{1}{\sqrt[4]{g}}
$$

as the system operator. The operator $\vec{D}_{2}$ is hermitian only for the inner product and the hermitian conjugate defined by

$$
\begin{gathered}
\tilde{f} \equiv \sqrt[4]{g} f \\
<\tilde{f}_{1} \mid \tilde{f}_{2}>\equiv \int d^{2} x \tilde{f}_{1}^{\dagger}(x) \tilde{f}_{2}(x)=\int d^{2} x \sqrt{g} f_{1}^{\dagger}(x) f_{2}(x) \\
<\vec{D}_{2} \tilde{f}_{1}\left|\tilde{f}_{2}>=<\tilde{f}_{1}\right| \vec{D}_{2}^{\dagger} \tilde{f}_{2}>
\end{gathered}
$$

In contrast with the inner product (7) in Sect.2, the dilaton factor $\exp (-2 \phi)$ does not appear in the above definition. The factor appears in the operator $\vec{D}_{2}$. As in the Sect.2, the pathintegral measure must be taken as

$$
\begin{gathered}
\vec{D}_{2} v_{n}=\sigma_{n} v_{n} \quad, \quad<v_{n} \mid v_{m}>=\int d^{2} x v_{n}^{\dagger} v_{m}=\delta_{n m} \\
\tilde{f}=\sum_{n} b_{n} v_{n} \quad, \quad b_{n} \equiv<v_{n} \mid \tilde{f}>\quad, \\
\exp \Gamma_{2}[g, \phi]=\int d \mu_{2}(f ; g, \phi) \exp S_{m}^{2}[f ; g, \phi] \quad, S_{m}^{2}=\frac{1}{2} \int d^{2} x \tilde{f} \vec{D}_{2} \tilde{f} \\
d \mu_{2}(f ; g, \phi)=\prod_{n} d b_{n} \equiv \mathcal{D} \tilde{f} .
\end{gathered}
$$


The same procedure as given in Sect.2 leads to the Weyl anomaly, using the measure defined by (20),

$$
\begin{gathered}
T_{2}=\lim _{t \rightarrow+0} \sum_{n} v_{n}^{\dagger}(x) v_{n}(x) \mathrm{e}^{-\sigma_{n} t}=\lim _{t \rightarrow+0} \sum_{n}<n\left|x>\mathrm{e}^{-\vec{D}_{2} t}<x\right| n> \\
=\lim _{t \rightarrow+0} \sum_{n} \operatorname{tr}<x\left|n>\mathrm{e}^{-\vec{D}_{2} t}<n\right| x>=\lim _{t \rightarrow+0} \operatorname{tr}<x\left|\mathrm{e}^{-\vec{D}_{2} t}\right| x>=\lim _{t \rightarrow+0} \operatorname{tr} G_{2}(x, x ; t) \\
G_{2}(x, y ; t) \equiv<x\left|\mathrm{e}^{-\vec{D}_{2} t}\right| y>
\end{gathered}
$$

We note the anomaly formula (16) can not be applied for $\vec{D}_{2}$ because of the dilaton factor $\exp (-2 \phi)$ in front of the second derivative term. We evaluate $G_{2}(x, x ; t)$ in the following two steps.

i) The flat space

In the case of the flat space, $g_{\mu \nu}=\delta_{\mu \nu}, \vec{D}_{2}$ reduces to

$$
\vec{D}_{2}=\mathrm{e}^{-2 \phi}\left(-\partial^{2}+2 \partial_{\mu} \phi \cdot \partial_{\mu}\right) .
$$

Now we consider the weak field case of the dilaton: $|\phi| \ll 1$. We fix the anomaly in a first few orders with respect to $\phi . \vec{D}_{2}$ is expressed as

$$
\begin{gathered}
\vec{D}_{2}=-\partial^{2}-W_{\mu \nu} \partial_{\mu} \partial_{\nu}-N_{\mu} \partial_{\mu}-M, \\
W_{\mu \nu}=\left(\mathrm{e}^{-2 \phi}-1\right) \delta_{\mu \nu}=\left(-2 \phi+2 \phi^{2}+\mathrm{O}\left(\phi^{3}\right)\right) \delta_{\mu \nu}, \\
N_{\mu}=-2 \mathrm{e}^{-2 \phi} \partial_{\mu} \phi=-2(1-2 \phi) \partial_{\mu} \phi+\mathrm{O}\left(\phi^{3}\right), \\
M=0,
\end{gathered}
$$

where $W_{\mu \nu}, N_{\mu}$ and $M$ are introduced for the general background fields and their explicit content, in the present case, are given above. From the definition of the heat-kernel (15), $G_{2}$ satisfy

$$
\left(\frac{\partial}{\partial t}-\partial^{2}\right) G_{2}(x, y ; t)=\left(W_{\mu \nu} \partial_{\mu} \partial_{\nu}+N_{\mu} \partial_{\mu}+M\right) G_{2}(x, y ; t),
$$

which is used to solve the heat-kernel perturbatively with respect to the fields in the right-hand side $(W, N, M)$. The 1 -st order result of $\left.G_{2}(x, x ; t)\right|_{t^{0}}$ was obtained in $(27)$ of 13 as

$$
\frac{1}{4 \pi}\left(-\frac{1}{12} \partial^{2} W_{\mu \mu}+\frac{1}{3} \partial_{\mu} \partial_{\nu} W_{\mu \nu}-\frac{1}{2} \partial_{\mu} N_{\mu}+M\right)
$$

This formula gives

$$
\frac{1}{4 \pi}\left(\frac{2}{3} \partial^{2} \phi-\frac{4}{3}(\partial \phi)^{2}\right)
$$

The 2nd order formula is similarly obtained as 22

$$
\begin{gathered}
\frac{1}{4 \pi}\left\{\frac{1}{48} \partial_{\mu} W_{\lambda \lambda} \cdot \partial_{\mu} W_{\sigma \sigma}-\frac{1}{12} \partial_{\mu} W_{\mu \nu} \cdot \partial_{\nu} W_{\lambda \lambda}-\frac{1}{4} \partial_{\mu} W_{\mu \nu} \cdot \partial_{\lambda} W_{\lambda \nu}\right. \\
\left.+\frac{1}{24} \partial_{\mu} W_{\lambda \sigma} \cdot \partial_{\mu} W_{\lambda \sigma}+\frac{1}{12} \partial_{\mu} W_{\nu \lambda} \cdot \partial_{\lambda} W_{\mu \nu}-\frac{1}{4} N_{\mu} N_{\mu}+\frac{1}{2} \partial_{\mu} W_{\mu \nu} \cdot N_{\nu}\right\} \\
+(W \partial \partial W, W \partial N, W M \text { terms })
\end{gathered}
$$


This formula gives, for the present model,

$$
\frac{1}{4 \pi} \times \frac{1}{3}(\partial \phi)^{2}
$$

Therefore we have

$$
q_{2}=\frac{1}{4 \pi}\left(-\frac{4}{3}+\frac{1}{3}\right)=-\frac{1}{4 \pi} \quad, \quad q_{3}=\frac{1}{4 \pi} \times \frac{2}{3} .
$$

ii) Vanishing Dilaton

In the case of vanishing dilaton, $\phi=0, \vec{D}_{2}$ reduces to $-\sqrt[4]{g} \nabla^{2} \frac{1}{\sqrt[4]{g}}$, and the Weyl anomaly is well known ( for example, see (29) of [13]).

$$
q_{1}=-\frac{1}{24 \pi}
$$

We note, in (30), that the value of $q_{3}$ differs from the result in Sect.2[23] whereas $q_{2}$ is the same. The $q_{3}$ value agrees with (16) of Ref. 21] with the choice $\psi(\phi)=0$ (trivial measure) and $\varphi(\phi)=\phi$ in the notation of the reference where the zeta-function regularization is taken.

\section{Discussion and Conclusion}

Generally the way to analyse a quantum field theory using some background (external) fields is quite popular in the theoretical physics. A systematic approach is called the background field method [24, 25] or the effective action approach[26]. The anomaly formulae (16), (26) and (28) (or its 4 dim version in [13, 19]) are based on the formalism. It should be noted that the anomaly formula is different from the counter-term formula in that it is definite including total derivative terms.

Taking the heat-kernel regularization, we have analysed the Weyl anomaly with special interst in the freedom of the choice of the system operator, which is caused by the arbitrariness of the total divergence. The operator $\vec{D}_{2}$ of Sect.3 was taken in [2], whereas $\vec{D}_{1}$ of Sect.2 was essetially taken in [6]. Although they are related by the total divergence, the corresponding Weyl anomalies become different because of the measure difference. (From this viewpoint, it is considered that the $a$-independence of the Weyl anomaly in Sect.2 shows that the measure difference due to the first derivative term do not influence the Weyl anomaly.) The most natural measures are fixed for each case from the requirement of the hermiticity of the system operators.

Let us compare the present situation with the case of the non-Abelian anomaly 27, 14, 15], where typically two types of anomalies exist, i.e., the consistent anomaly and the covariant anomaly. In Ref. [19], they are treated in the present approach. For the system of the chiral (right) fermion coupled with the gauge field in 4 dim space: $\mathcal{L}_{R}=-\bar{\psi} \gamma^{\mu}\left(\partial_{\mu}-i \frac{1+\gamma_{5}}{2} R_{\mu}\right) \psi \equiv-\bar{\psi} \not D_{R+} \psi$, the consistent anomaly is obtained by the choice $\vec{D}_{\text {cons }}=-\not_{R+} \not D_{R+}$ (non-hermitian), and the covariant one by $\vec{D}_{c o v}=\not D_{R+}^{\dagger} \not D_{R+}$ (hermitian). 
$\vec{D}_{\text {cons }}$ is directly related with the effective action as $\sqrt{\operatorname{det} \vec{D}_{\text {cons }}}=\int \mathcal{D} \psi \mathcal{D} \bar{\psi} \int^{\int \mathcal{L}_{R}}$. On the other hand, $\vec{D}_{\text {cov }}$ does not have such a direct relation: $\operatorname{det} \vec{D}_{\text {cov }}=\left|\int \mathcal{D} \psi \mathcal{D} \bar{\psi} \mathrm{e}^{\int \mathcal{L}_{R}}\right|^{2}$. This corresponds to the fact that the consistent anomaly satisfies the consistency condition derived from the "formal" (without touching on the regularization problem) effective action analysis, whereas the covariant one does not. In the Fujikawa's standpoint, however, the anomaly is not defined by the "formal" effective action analysis. It originates from the regularization of $\delta^{n}(x-y)$ (in $n$ dim space) which appears in the Jacobian at the change of the integration variable fields. The anomaly comes from the integration measure at the regularized level. In the heat-kernel regularization, we take

$\delta^{n}(x-y) \equiv \lim _{t \rightarrow+0}<x\left|\mathrm{e}^{-\vec{D} t}\right| y>$ where the connection with the system is provided by the operator $\vec{D}$. This "flexibility" is one of the advantages of the Fujikawa's approach. In this standpoint, the regularization dependence in the Weyl anomaly found in the present paper is similar to that in the non-Abelian anomaly except the following points.

1. $\vec{D}_{1}$ and $\vec{D}_{2}$ in the Weyl anomaly case are related by the total divergences, whereas $\vec{D}_{\text {cons }}$ and $\vec{D}_{\text {cov }}$ in the non-Abelian anomaly case are not.

2. Corresponding to the above item, in the Weyl anomaly case the two choices of the operators give the difference only in a part of the whole anomaly, that is, the trivial term. In the non-Abelian anomaly case, the two choices give totally different anomalies.

The heat-kernel calculation in Sect.3 is equivalent to the generalized zeta function $\left(\zeta_{\vec{D}_{2}}(s)\right)$ analysis taken in [2]. It is well known $\zeta_{\vec{D}_{2}}(s)$ is related with $G_{2}(x, x ; t)$ of (22) as

$$
\begin{gathered}
Y(t) \equiv \int d^{2} x G_{2}(x, x ; t)=\sum_{n} \mathrm{e}^{-\sigma_{n} t}, \\
\zeta_{\vec{D}_{2}}(s)=\frac{1}{\Gamma(s)} \int_{0}^{\infty} Y(t) t^{s-1} d t=\sum_{n} \sigma_{n}^{-s} .
\end{gathered}
$$

Technically Ref. [2] evaluated $\left.\zeta_{\vec{D}_{2}}(s)\right|_{s=0}$, whereas this paper has evaluated $\left.G_{2}(x, x ; t)\right|_{t=0}$.

We have treated the metric and the dilaton as background (classical) fields. Their quantum effects can be straightforwardly taken into account by following the background formalism. (In fact they are evaluated in [6, 07, 8].) At present, however, it looks that the correct quantum treatment of the metric and the dilaton will be given by the string theory.

The Weyl anomaly is a key factor in the Hawking radiation phenomenon. In the present case of 2 dimension, the energy momentum tensor $T_{\mu \nu}$ is determined by the conformal anomaly and the conservation equation with an appropriate boundary condition.

$$
\begin{gathered}
\sqrt{g} g^{\mu \nu} T_{\mu \nu}=\sqrt{g}\left(-\frac{1}{24 \pi} R-\frac{1}{4 \pi}(\nabla \phi)^{2}+q_{3} \nabla^{2} \phi\right), \\
\nabla^{\mu} T_{\mu \nu}=0
\end{gathered}
$$

The different values of $q_{3}$ should not affect physical quantities. If the above equations are right, they show the energy momentum tensor itself depends on $q_{3}$. It seems to give some 
trouble about the physical interpretation of the energy momentum tensor and the radiation amplitude. We should notice here the following points: 1) we do not take into account the contribution of the quatum effect of the dilaton and the graviton; 2) the $q_{3}$ term in (33) can be freely changed by introducing a finite counterterm, $\phi R$, in the gravity-dilaton lagrangian in advance as noted in (5). This problem looks to involve graviton-dilaton dynamics and "renormalization" of its lagrangian. In the present analysis of perturbative quantization of matter fields, we have no control over such a problem. Although the above problem is beyond the scope of the present work, it is highly interesting that the black hole physics is not closed within the quantum matter-field theory on the background curved space, but open to the quantum gravity problem through the Weyl anomaly.

\section{Acknowledgement}

The author thanks Prof.S.D.Odintsov and Prof.S.Nojiri for informing their stimulating results and discussions about this subject. He also thanks Prof. K.Fujikawa for an encouraging comment and some corrections in the original manuscript.

\section{References}

[1] C.G.Callan, S.B.Giddings,J.A.Harvey and A.Strominger,Phys.Rev.D45, 1005(1992).

[2] R.Bousso and S.W.Hawking,Phys.Rev.D56,7788(1997) hep-th/9705236.

[3] J.G.Russo,L.Susskind and L.Thorlacius,Phys.Lett.B292,13(1992).

[4] A.Bilal and C.Callan,Nucl.Phys.B394,73(1993).

[5] E.Elizalde,S.Naftulin and S.D.Odintsov,Phys.Rev.D49,2852(1994).

[6] S.Nojiri and S.D.Odintsov,Mod.Phys.Lett.A12,2083(1997), hep-th/9706009.

[7] A.Miković and V.Radovanović,FTUV/97-35,IFIC/97-34, hep-th/9706066, "One-loop Effective Action for Spherical Scalar Field Collapse"

[8] S.Nojiri and S.D.Odintsov,NDA-FP-35,hep-th/9706143, Phys.Rev.D to appear, "Trace anomaly and induced effective Action for 2D and 4D dilaton coupled scalars".

[9] L.Bonora,P.Pasti and M.Bregola,Class.Quantum.Grav.3,635(1986).

[10] S.Deser and A.Schwimmer,Phys.Lett.B309,279(1993). 
[11] T.Arakelyan,D.R.Karakhanyan,R.P.Manvelyan and R.L.Mkrtchyan, Phys.Lett.B353,52(1995).

[12] D.R.Karakhanyan,R.P.Manvelyan and R.L.Mkrtchyan, Mod.Phys.Lett.A11,409(1996).

[13] S.Ichinose and N.Ikeda,Phys.Rev.D53,5932(1996).

[14] K.Fujikawa,Phys.Rev.Lett.42,1195(1979);441733(1980); Phys.Rev.D21,2848(1980); D22,1499(E)(1980); D23,2262(1981).

[15] For review, K.Fujikawa,Lecture in Quantum Gravity and Cosmology, Kyoto Summer Inst.1985,ed.by Sato and Inami,World Scientific :106(1985).

[16] As for the relation of the present approach 3) to the approach 1), see Sect.4.

[17] We do not take the 'parity' symmetry $\phi \rightarrow-\phi$, which appears in the S-duality in the string theory. That demands $q_{3}=0$, which is inconsistent with the explicit (perturbative) result.

[18] K.Fujikawa,Nucl.Phys.B226,437(1983).

[19] S.Ichinose and N.Ikeda,DAMTP/96-87, hep-th/9610136, "Gauge Symmetry of the Heat Kernel and Anomaly Formulae".

[20] The result $q_{3}=1 / 4 \pi$ also agrees with (16) of Ref. 21] with $\varphi(\phi)=\psi(\phi)=\phi$ in its notation.

[21] W.Kummer,H.Liebl and D.V.Vassilevich,Mod.Phys.Lett.A12,2683(1997) hep-th/9707041.

[22] Eq.(28) is obtained by evaluating eq.(25) of Ref. 13 taking $n=2$ there.

[23] $q_{3}$ of (31) differs from [2] too. Note that their derivation of $q_{3}$ is ambiguous as mentioned in Sect.1. Because the calculational circumstance (regularization, operator, measure) is essentially the same (see Sect.4), we expect their value of $q_{3}$ should coincide with (30).

[24] B.S.DeWitt,Phys.Rev.162,1195(1967);162,1239(1967).

[25] G.'tHooft,Nucl.Phys.B62,444(1973).

[26] I.L.Buchbinder,S.D.Odintsov and I.L.Shapiro,Effective Action in Quantum Gravity,IOP Pub.,1992.

[27] W.A.Bardeen,Phys.Rev.184,1848(1969) 